\title{
Study of Baryon Spectroscopy Using a New Potential Form
}

\author{
L. I. Abou-Salem \\ Physics Department, Faculty of Science, Benha University, Benha, Egypt \\ Correspondence should be addressed to L. I. Abou-Salem; loutfy.abousalem@fsc.bu.edu.eg
}

Received 23 May 2014; Revised 1 August 2014; Accepted 17 August 2014; Published 10 September 2014

Academic Editor: Elias C. Vagenas

Copyright (C) 2014 L. I. Abou-Salem. This is an open access article distributed under the Creative Commons Attribution License, which permits unrestricted use, distribution, and reproduction in any medium, provided the original work is properly cited. The publication of this article was funded by SCOAP ${ }^{3}$.

In the present work, the nonrelativistic quark model is applied to study baryon systems, where the constituent quarks are bound by a suitable hyper central potential. We proposed a new phenomenological form of the interaction potential, digamma-type potential. Using the Jacobi coordinates, the three-body wave equation is solved numerically to calculate the resonance states of the $N, \Delta, \Lambda$, and $\Sigma$ baryon systems. The present model contains only two adjustable parameters in addition to the quark masses. Our theoretical calculations are compared to the available experimental data and Cornell potential results. The description of the spectrum shows that the ground states of the considered light and strange baryon spectra are in general well reproduced.

\section{Introduction}

Hadron spectroscopy is very important to study its structures and the nature of the interaction forces between its constituents. In the previous works [1-5], the heavy meson spectroscopy is studied by using different potential models. Many authors have been studying the baryon spectroscopy. An analytical solution was provided in case of harmonic and anharmonic potentials for a system consists of three identical particles $[6,7]$. Some authors have used the Cornell potential (Coulomb-type plus linear term) to study the resonance states of $N$ and $\Delta$ baryons [8-14]. The harmonic oscillator potential has been used to study the interaction between three identical particles [15]. In the present work, the digamma-function is used as the interaction potential between constituent quarks of baryon systems. The threebody wave equation is solved, numerically, by using the Jacobi method to calculate the resonance state masses of $N, \Delta, \Lambda$, and $\Sigma$ baryons. These baryons consist of identical or nonidentical quarks. Our theoretical results will be investigated in Section 3.

\section{The Used Model}

The nonrelativistic Schroedinger equation for a system consists of three particles is given as

$$
\begin{aligned}
& {\left[-\frac{\hbar^{2}}{2 m_{1}} \nabla_{r_{1}}^{2}-\frac{\hbar^{2}}{2 m_{2}} \nabla_{r_{2}}^{2}-\frac{\hbar^{2}}{2 m_{3}} \nabla_{r_{3}}^{2}+V_{12}\left(r_{12}\right)\right.} \\
& \left.+V_{23}\left(r_{23}\right)+V_{31}\left(r_{31}\right)\right] \Psi\left(r_{1}, r_{2}, r_{3}\right) \\
& =E \Psi\left(r_{1}, r_{2}, r_{3}\right),
\end{aligned}
$$

where $m_{1}, m_{2}$, and $m_{3}$ are the three-quark masses and $r_{i j}$ is the relative separation vector.

In the quark model, a baryon is a three-body bound state made of quarks. The mathematical description of a threebody system is more complicated than that of a two-body system. Several methods have been used by different authors to solve three-body problems $[11,15]$. In order to describe the baryon as a bound state of three constituent quarks, we define 
the configuration of three particles by the center of mass, $R$, and two Jacobi coordinates $\rho$ and $\lambda$ as

$$
\begin{array}{r}
\vec{R}=\frac{m_{1} \overrightarrow{r_{1}}+m_{2} \overrightarrow{r_{2}}+m_{3} \overrightarrow{r_{3}}}{M}, \\
\vec{\rho}=c_{1}\left(\overrightarrow{r_{1}}-\overrightarrow{r_{2}}\right), \\
\vec{\lambda}=c_{2}\left(\frac{m_{1} \overrightarrow{r_{1}}+m_{2} \overrightarrow{r_{2}}}{M^{\prime}}-\overrightarrow{r_{3}}\right),
\end{array}
$$

where $M=m_{1}+m_{2}+m_{3} ; M^{\prime}=m_{1}+m_{2} ; c_{1}$ and $c_{2}$ are constants.

Using natural units ( $\hbar=C=1$ ) and letting the coefficients of $\nabla_{\rho}^{2}$ and $\nabla_{\lambda}^{2}$ be equal to $A$, therefore, (1) can be separated into the following two equations:

$$
\begin{gathered}
-\frac{1}{2 M} \nabla_{R}^{2} \Psi_{C . M}(R)=E_{1} \Psi_{C . M}(R), \\
{\left[-A\left(\nabla_{\rho}^{2}+\nabla_{\lambda}^{2}\right)+V(x)\right] \Psi(\vec{\rho}, \vec{\lambda})=E_{2} \Psi(\vec{\rho}, \vec{\lambda}),}
\end{gathered}
$$

where

$$
E=E_{1}+E_{2}, \quad A=\frac{c_{1}^{2}}{2 \mu_{12}}=\frac{c_{2}^{2}}{2 \mu_{12,3}},
$$

where

$$
\mu_{12}=\frac{m_{1} m_{2}}{m_{1}+m_{2}}, \quad \mu_{12,3}=\frac{m_{3}\left(m_{1}+m_{2}\right)}{m_{1}+m_{2}+m_{3}} .
$$

The wave function $\Psi(\vec{\rho}, \vec{\lambda})$ can be expressed as $[8,16,17]$

$$
\Psi(\vec{\rho}, \vec{\lambda})=\sum_{\gamma} \Psi_{\gamma}(x) Y_{\gamma}(\Omega)
$$

where $\Psi_{\gamma}(x)$ and $Y_{\gamma}(\Omega)$ are the hyper radial part of the wave function and the hyper spherical harmonic function, respectively. The symbol $\gamma$ is called the grand angular quantum number and is given by

$$
\gamma=2 v+l_{\rho}+l_{\lambda}
$$

where $l_{\rho}$ and $l_{\lambda}$ are the angular momenta associated with the $\rho$ and $\lambda$ variables and $v$ is a nonnegative integer number.

The hyperradius $x$ is given as

$$
x=\sqrt{\rho^{2}+\lambda^{2}}=\sqrt{\frac{1}{3}\left(r_{12}^{2}+r_{23}^{2}+r_{31}^{2}\right)} .
$$

The potential $V(x)$ can be assumed to depend on the hyperradius $x$ and $e$ space wave function is factored similar to the central potential [8].

Equation (4) can be rewritten as

$$
\begin{gathered}
\frac{d^{2} \Psi_{\gamma}(x)}{d x^{2}}+\frac{D-1}{x} \frac{d \Psi_{\gamma}(x)}{d x}-\frac{L_{(\Omega)}^{2}}{x^{2}} \Psi_{\gamma}(x) \\
+\frac{1}{A}(E-V(x)) \Psi_{\gamma}(x)=0,
\end{gathered}
$$

where $D$ represents the dimension of the $\vec{x}$ and $L_{(\Omega)}^{2}$ is the angular momentum operator whose eigenfunctions are $[6,9$, 11]

$$
L_{(\Omega)}^{2} Y_{\gamma}=-\gamma(\gamma+D-2) Y_{\gamma}
$$

In the present work, the interaction potential is assumed as digamma-type as follows:

$$
\begin{aligned}
V(x) & =C \frac{\Gamma^{\prime}(x)}{\Gamma(x)} \\
& =C \int_{0}^{\infty}\left(\frac{e^{-t}}{t}-\frac{e^{-x t}}{1-e^{-t}}\right) d t,
\end{aligned}
$$

where $C$ is an adjustable parameter and $\Gamma(x)$ is the gammafunction.

Now, applying the following transformation

$$
\Psi_{\gamma}(x)=\frac{u_{\gamma}(x)}{x^{(D-1) / 2}}
$$

(10) can be rewritten as

$$
\begin{aligned}
& {\left[\frac{d^{2}}{d x^{2}}+\frac{1}{A}(E-V(x)-A\right.} \\
& \left.\left.\quad \times\left(\frac{(D-1)(D-3)}{4 x^{2}}+\frac{\gamma(\gamma+D-2)}{x^{2}}\right)\right)\right] \\
& \quad \times u_{\gamma}(x)=0
\end{aligned}
$$

With the new boundary conditions,

$$
u_{\gamma}(0)=u_{\gamma}(\infty)=0 \text {. }
$$

Using

$$
\begin{gathered}
\lambda=\frac{E}{A}, \\
\varphi(x)=V(x)+\frac{(D-1)(D-3)}{4 x^{2}}+\frac{\gamma(\gamma+D-2)}{x^{2}} .
\end{gathered}
$$

Equation (12) can be rewritten as

$$
\frac{d^{2} u_{\gamma}(x)}{d x^{2}}+(\lambda-\varphi(x)) u_{\gamma}(x)=0 .
$$

To transform the range of $x$ from $(\infty, 0)$ to $(0,1)$, I introduce the dimensionless variables, $g, g=1 /\left(1+\left(x / x_{0}\right)\right)$, and assume that

$$
\rho(g)=g u(g)
$$

where $x_{o}=1 \mathrm{GeV}^{-1}$.

Equation (18) can be rewritten as

$$
\frac{d^{2} \rho(g)}{d g^{2}}+\left[\frac{x_{0}^{4}}{g^{4}}\right](\lambda-\varphi(g)) \rho(g)=0 .
$$


The new boundary conditions are given as

$$
\rho(0)=\rho(1)=0 \text {. }
$$

To transform (20) into a matrix form, one can divide the range of $(g)$ from $(0,1)$ into $(n+2)$ points with equal interval, $h$, labeled by subscript $(J)$.

The new form of the boundary conditions is given as

$$
\rho_{n+1}=\rho_{0}=0 .
$$

Using the finite difference approximation [19], the second derivative term in (20) is given as

$$
\begin{aligned}
\frac{d^{2} \rho(g)}{d g^{2}}=\frac{1}{12 h^{2}}[ & -\rho_{J-2}+16 \rho_{J-1}-30 \rho_{J} \\
& \left.+16 \rho_{J+1}-\rho_{J+2}\right]+O\left(h^{4}\right),
\end{aligned}
$$

where the term $O\left(h^{4}\right)$ represents the expected error.

Using the symmetry properties of the radial wave function, one can assume that [15]

$$
\begin{gathered}
\rho_{-1}=(-1)^{\gamma} \rho_{1}+O\left(h^{2}\right) ; \\
\rho_{n+2}=(-1)^{\gamma+1} \rho_{n}+O\left(h^{3}\right) .
\end{gathered}
$$

Using (21), (22), and (23) in (18) and taking $x_{0}=1 \mathrm{GeV}^{-1}$, one gets

$$
\begin{gathered}
\left(\rho_{J-2}-16 \rho_{J-1}+30 \rho_{J}-16 \rho_{J+1}+\rho_{J+2}\right) \\
+\frac{12 h^{2}}{(J h)^{4}}(\varphi(J h)-\lambda) \rho_{J}=0
\end{gathered}
$$

This equation is rewritten in the following matrix form:

$$
(A-\lambda I) \rho=0,
$$

where $A$ is $n \times n$ symmetric matrix and $I$ is $n \times n$ unitary diagonal matrix. Using the Jacobi method [20, 21], (26) can be solved numerically and the eigenvalues, $E$, are determined. The nonrelativistic resonance mass, $M$, for each state is given by

$$
M=m_{1}+m_{2}+m_{3}+E .
$$

\section{Results and Discussions}

In the present work, the spin-spin interaction is not considered and we deal with the spin averaged stats. The predicted values of the $N, \Delta, \Lambda$, and $\Sigma$ resonance masses are calculated through solving (26) numerically using the Jacobi method. The present potential form is proposed as the analytical form of the digamma-function; see (12) and (13). The $\chi^{2}$-test is used to determine the parameter values

$$
\chi^{2}=\frac{1}{N} \sqrt{\sum_{i=1}^{n}\left(\frac{\left(M_{i}^{\text {theo }}-M_{i}^{\exp }\right)}{e_{i}}\right)^{2}},
$$

TABLE 1: The values of the parameters which are taken in our calculations.

\begin{tabular}{lc}
\hline Parameters & Values \\
\hline$m_{u}$ & $0.035 \pm 0.005 \mathrm{GeV}$ \\
$m_{d}$ & $0.035 \pm 0.005 \mathrm{GeV}$ \\
$m_{s}$ & $0.092 \pm 0.008 \mathrm{GeV}$ \\
$A$ & $0.40 \pm 0.04 \mathrm{GeV}^{-1}$ \\
$C$ & $0.85 \pm 0.08 \mathrm{GeV}$ \\
\hline
\end{tabular}

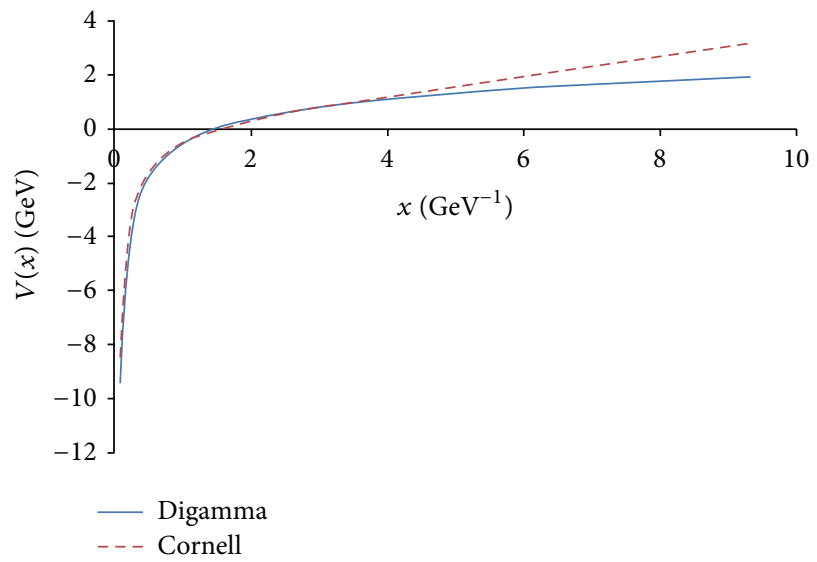

Figure 1: The digamma-type and Cornell potentials versus the hyperradius $x$.

where $N$ is the total number of states, $M$ is the resonance mass, and $e_{i}$ is the experimental error in the $i$ th State. The parameter values which are used in the present calculations are given in Table 1. The uncertainties in these parameters may be due to relativistic effects and ignoring the spin interaction terms.

The behavior of the digamma-type and Cornell potentials versus the hyperradius, $x$, is shown in Figure 1. From this figure one notices that the suggested potential behaves nearly like Cornell potential at small $x$ (one gluon exchange part) and the Cornell potential is more confined than the digamma-type potential at large $x$.

Table 2 contains the present calculations of $N$ resonance states using both digamma-function potential and a Coulomb like hyper central potential plus the linear term (Cornell Model [10]) in comparison to the experimental values [18]. From this table, one notices that the observed seven experimental states are calculated using the suggested potential form while the results of Cornell potential are 4 states only and the present calculations give more satisfied agreement with the experimental data.

Table 3 contains the present calculations of $\Delta$ baryon states and Cornell potential results [10] in comparison to the available experimental data [18]. From this figure one notices that the observed 5 states are calculated using the present potential form while the calculated states in [10] are three states only. In case of $N$ and $\Delta$ baryons, one can notice that the calculated resonance masses using the digamma-type 
TABLE 2: The present calculations of $N$ baryon states in comparison to the Cornell potential results [10] and the experimental data [18] in $(\mathrm{MeV})$.

\begin{tabular}{lcccc}
\hline Baryon state & $M_{\text {exp }}$ ref. [18] & $\gamma$ & Ref. [10] & Present calculations \\
\hline$N$ (938) P11 & $938.272013 \pm 0.000023$ & 938 & 938.25 \\
$N$ (1440) P11 & $1445 \pm 25$ & 0 & 1463 & 1448.43 \\
$N$ (1710) P11 & $1710 \pm 30$ & 1752 & 1702.24 \\
\hline$N$ (1535) S11 & $1535 \pm 10$ & 1524 & 1544.67 \\
$N$ (1905) S11 & 1905 & 1 & - & 1902.34 \\
$N$ (2090) S11 & $2150 \pm 50$ & - & 2130.87 \\
\hline
\end{tabular}

TABLE 3: The present calculations of $\Delta$ baryon states in comparison with the Cornell potential results [10] and the experimental data [18] in $(\mathrm{MeV})$.

\begin{tabular}{lcccc}
\hline Baryon state & $M_{\text {exp }}$ ref. [18] & $\gamma$ & Ref. [10] & Present calculations \\
\hline$\Delta$ (1232) P33 & $1232 \pm 1$ & 0 & 1232 & 1233.24 \\
$\Delta$ (1600) P33 & $1625 \pm 75$ & & 1727 & 1594.57 \\
\hline$\Delta$ (1620) S31 & $1630 \pm 30$ & & 1573 & 1612.95 \\
$\Delta$ (1900) S31 & 1900 & 1 & - & 1896.32 \\
$\Delta(2150)$ S31 & 2150 & & - & 2160.73 \\
\hline
\end{tabular}

TABLE 4: The present calculations of $\Lambda$ baryon states in comparison to the experimental data $[18]$ in $(\mathrm{MeV})$.

\begin{tabular}{lccc}
\hline Baryon state & $M_{\text {exp }}$ ref. [18] & $\gamma$ & Present calculations \\
\hline$\Lambda$ (1116) P01 & $1115.683 \pm 0.006$ & & 1116.85 \\
$\Lambda$ (1600) P01 & $1630 \pm 70$ & 0 & 1689.46 \\
$\Lambda$ (1810) P01 & $1800 \pm 50$ & & 1832.86 \\
\hline$\Lambda$ (1670) S01 & $1670 \pm 10$ & 1 & 1665.21 \\
$\Lambda$ (1800) S01 & $1785 \pm 65$ & & 1851.75 \\
\hline$\Lambda$ (1890) P03 & $1880 \pm 30$ & 2 & 1915.79 \\
\hline
\end{tabular}

TABLE 5: The present calculations of $\Sigma$ baryon states in comparison to the experimental data $[18]$ in $(\mathrm{MeV})$.

\begin{tabular}{lccc}
\hline Baryon state & $M_{\exp }$ ref. [18] & $\gamma$ & Present calculations \\
\hline$\sum(1193)$ P11 & $1192.642 \pm 0.024$ & & 1193.15 \\
$\Sigma(1660)$ P11 & $1660 \pm 30$ & 0 & 1629.47 \\
$\sum(1880)$ P11 & 1880 & & 1868.13 \\
\hline$\sum(1620)$ S11 & 1620 & 1 & 1617.39 \\
$\sum(1750)$ S11 & $1765 \pm 35$ & & 1809.35 \\
\hline
\end{tabular}

potential agree more with the experiment than the Cornell potential model results [10].

The present calculations of $\Lambda$ and $\Sigma$ baryon states compared with the experimental values [21] are given in Tables 4 and 5 . One notices that the suggested potential model can reproduce the experimental results of the ground states of $\Lambda$ and $\Sigma$ baryon systems.

\section{Conclusions}

A nonrelativistic quark model is used to study the spectra of the considered baryon systems ( $N, \Delta, \Lambda$, and $\Sigma$ baryons).
Using the hyper central approach, we have simplified the three-body problem and solved the Schrodinger equation numerically to obtain the ground-state energy eigenvalues and eigenfunctions. In the present work, we proposed a new phenomenological form of the interaction potential, digamma-type potential, between the quark constituents to study baryon systems. The behavior of this analytical function may be the same as the quark-antiquark interaction inside quarkonium systems. The description of the spectrum shows that the light and strange baryon ground states are in general well reproduced. The suggested potential model may be used to describe the energies of the higher excited states and negative-parity resonances through introducing the spin interaction terms as perturbed terms. Finally, this approach is useful because it allows for predictions of baryon states without extended lattice calculations and provides useful information about the short-distance one gluon exchange and the long-distance confinement interactions that can be useful in understanding the nature of the quark-quark force generated by QCD.

\section{Conflict of Interests}

The author declares that there is no conflict of interests regarding the publication of this paper.

\section{References}

[1] L. I. Abou-Salem, "Mass spectra and leptonic decay widths of heavy quarkonia by using psi function," Journal of Physics G: Nuclear and Particle Physics, vol. 30, no. 10, pp. 1391-1399, 2004.

[2] L. I. Abou-Salem, "A systematic study on nonrelativistic quarkantiquark interactions," International Journal of Modern Physics A, vol. 20, no. 17, p. 4113, 2005.

[3] L. I. Abou-Salem, M. S. M. Nour El-Din, and M. M. Moussa, "A study on breit interaction through heavy mesons spectra," Turkish Journal of Physics, vol. 29, no. 2, pp. 69-77, 2005.

[4] C. Amsler, T. DeGrand, and B. Krusche, "Review of particle physics," Physics Letters B, vol. 667, no. 1-5, pp. 1-6, 2008.

[5] B. Metsch, "Quark model description of hadrons," AIP Conference Proceedings, vol. 717, no. 1, pp. 646-655, 2004.

[6] A. A. Rajabi, "A three-body force model for the harmonic and anharmonic oscillator," Iranian Journal of Physics Research, vol. 5, no. 2, p. 37, 2005.

[7] A. A. Rajabi, "Exact analytical solution of the schrödinger equation for an $\mathrm{N}$-identical body-force system," Few-Body Systems, vol. 37, no. 4, pp. 197-213, 2005.

[8] E. Santopinto, F. Iachello, and M. M. Giannini, "Exactly solvable models of baryon spectroscopy," Nuclear Physics A, vol. 623, no. 1-2, pp. 100-109, 1997.

[9] M. Ferraris, M. M. Giannini, M. Pizzo, E. Santopinto, and L. Tiator, "A three-body force model for the baryon spectrum," Physics Letters B, vol. 364, no. 4, pp. 231-238, 1995.

[10] M. M. Giannini, E. Santopinto, and A. Vassallo, "Hypercentral constituent quark model and isospin dependence," The European Physical Journal A, vol. 12, p. 447, 2001.

[11] I. M. Narodetskii and M. A. Trusov, "The doubly heavy baryons," Nuclear Physics B: Proceedings Supplements, vol. 115, pp. 20-23, 2003. 
[12] E. Santopinto, F. Iachello, and M. M. Giannini, "Nucleon form factors in a simple three-body quark model," European Physical Journal A, vol. 1, no. 3, pp. 307-315, 1998.

[13] M. M. Giannini, E. Santopinto, and A. Vassallo, "An overview of the hypercentral constituent quark model," Progress in Particle and Nuclear Physics, vol. 50, no. 2, pp. 263-272, 2003.

[14] R. Bijker, F. Iachello, and E. Santopinto, "Algebraic treatment of the hyper-Coulomb problem," Journal of Physics A, vol. 31, no. 45, pp. 9041-9054, 1998.

[15] E. Cuervo-Reyes, M. Rigol, and J. Rubayo-Soneira, "Hadron spectra from a non-relativistic model with confining harmonic potential," Revista Brasileira de Ensino de Fisica, vol. 25, no. 1, p. 18, 2003.

[16] J. S. Avery, "Harmonic polynomials, hyperspherical harmonics, and atomic spectra," Journal of Computational and Applied Mathematics, vol. 233, no. 6, pp. 1366-1379, 2010.

[17] J. L. Ballot and M. Fabre de la Ripelle, "Application of the hyperspherical formalism to the trinucleon bound state problems," Annals of Physics, vol. 127, no. 1, pp. 62-125, 1980.

[18] K. Nakamura, "Review of particle physics," Journal of Physics G: Nuclear and Particle Physics, vol. 37, no. 7, Article ID 075021, 2010.

[19] M. Abramomwitiz and I. A. Stegun, Handbook of Mathematical Functions, National Bureau of Standards U.S. Gpo, Washington, DC, USA, 1972.

[20] J. H. Mathews and K. K. Fink, Numerical Methods Using Matlab, 4th edition, 2004.

[21] A. R. Goulary and G. A. Watson, Computational Methods for Matrix Eigen Problems, John Wiley \& Sons, 1973. 

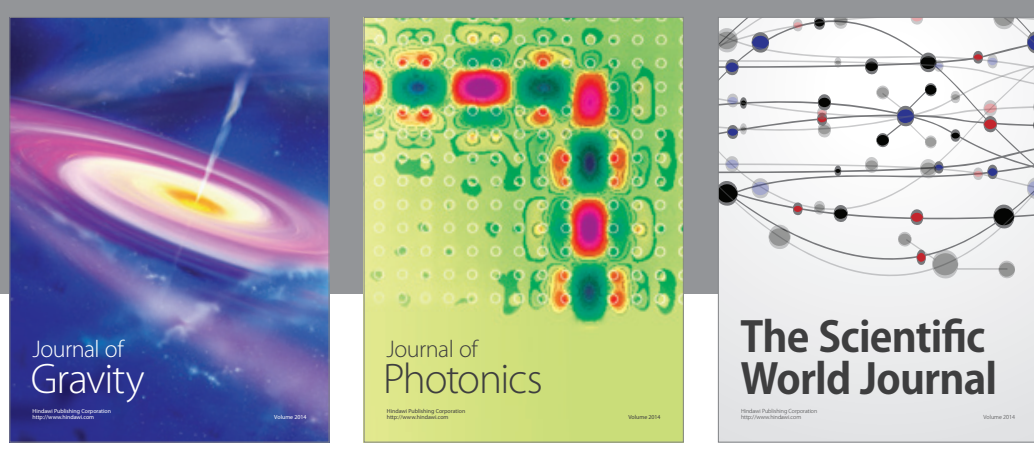

The Scientific World Journal
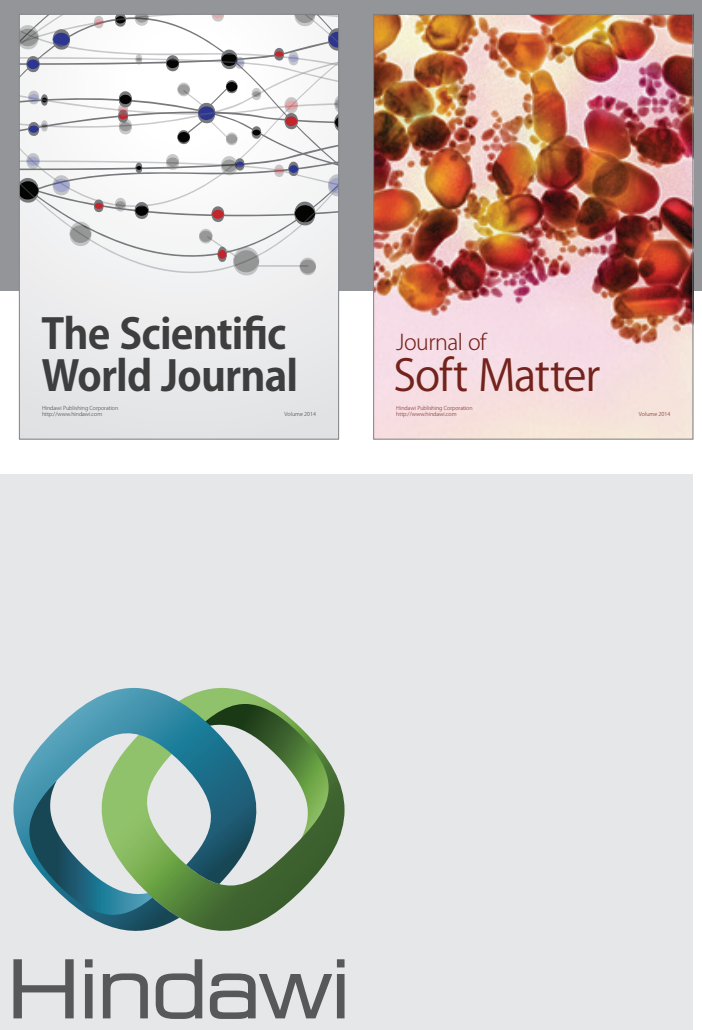

Submit your manuscripts at

http://www.hindawi.com

nternational Journal of

Statistical Mechanics
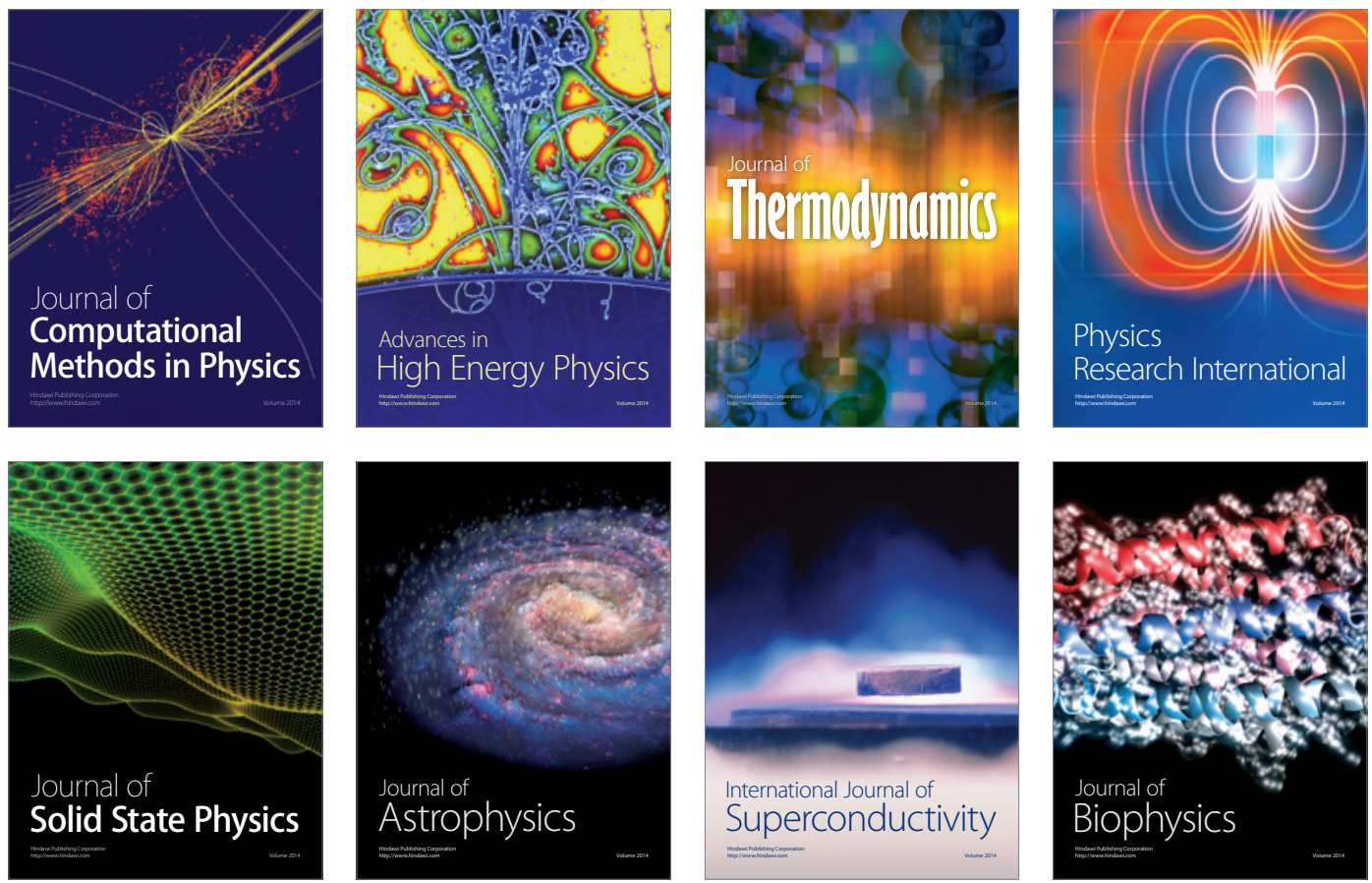
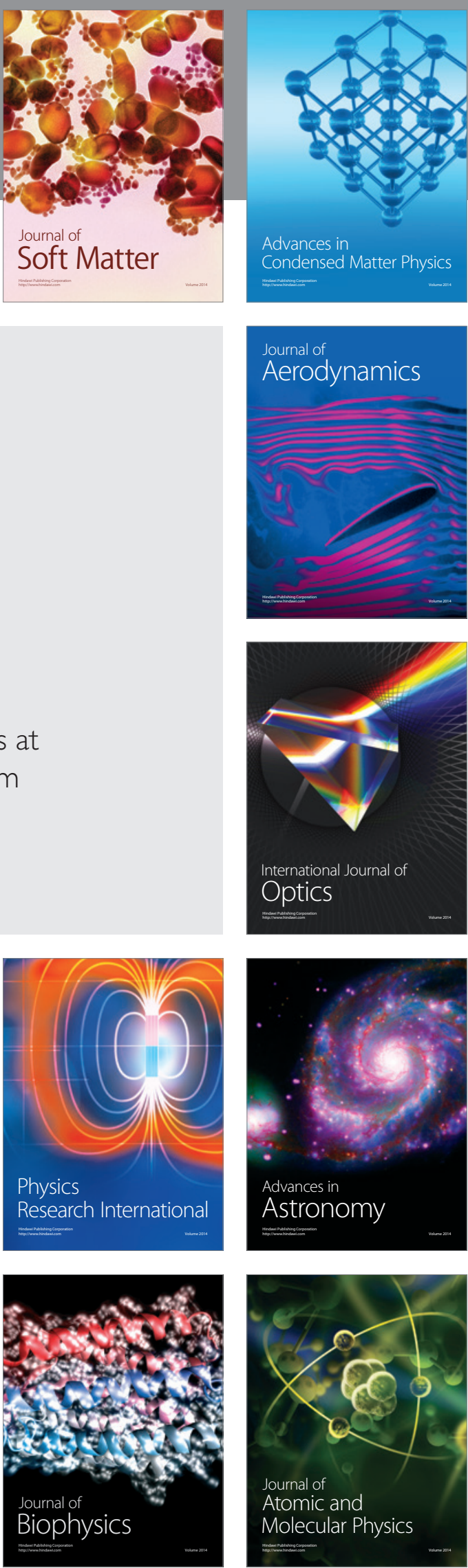